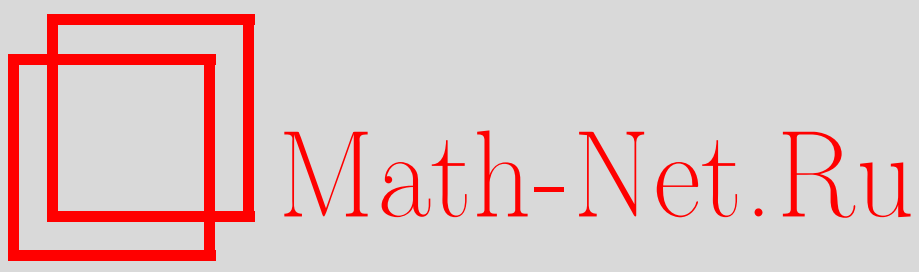

С. Х. Арансон, Е. В. Жужома, В. С. Медведев, О непрерывности геодезических каркасов потоков на поверхностях, Матем. сб., 1997, том 188, номер 7, 3-22

DOI: https://doi.org/10.4213/sm239

Использование Общероссийского математического портала Math-Net.Ru подразумевает, что вы прочитали и согласны с пользовательским соглашением http://www . mathnet.ru/rus/agreement

Параметры загрузки:

IP: 54.172 .240 .79

26 апреля 2023 г., 14:47:38 
УДК $517.917+513.9$

\author{
С.Х. Арансон, Е.В. Жужома, В.С. Медведев
}

\title{
О непрерывности геодезических каркасов потоков на поверхностях
}

\begin{abstract}
В статье для потоков на ориентируемой замкнутой поверхности $M_{g}$ большего рода (т.е. рода $g \geqslant 2$ ) строится специальное геодезическое распределение (геодезический каркас потока), состоящее из геодезических с теми же самьми асимптотическими направлениями, что и траектории потока, и которое является полным топологическим инвариантом иррациональных потоков на таких поверхностях. Рассматривается задача о зависимости геодезического каркаса от возмущения потока (или от параметра семейства потоков). Показьвается, что неприводимьй простейший иррациональный геодезический каркас потока непрерывно зависит от возмущения потока (что аналогично непрерывной зависимости иррационального числа вращения Пуанкаре от возмущения потока).
\end{abstract}

Библиография: 16 названий.

\section{Введение}

В статье каждому потоку из широкого класса потоков на ориентируемой замкнутой поверхности отрицательной эйлеровой характеристики ставится в соответствие геометрический объект - специальное геодезическое распределение (геодезический каркас потока), и исследуется поведение этого объекта при возмушениях потока. Геодезический каркас отражает асимптотическое поведение траекторий и полутраекторий потока и является его важной характеристикой (в особенности для потоков с нетривиально рекуррентньми траекториями, см. обзор [1]).

Важньм топологическим инвариантом потоков на двумерном торе $T^{2}$ является число вращения Пуанкаре, которое отражает асимптотическое поведение траекторий потока. Для минимальных потоков на торе число вращения является полньм топологическим инвариантом (с точностью до пересчета с помощью автоморфизма фундаментальной группы), иррационально и непрерьвно зависит от возмущения потока.

Любой поток на ориентируемой замкнутой поверхности $M_{g}$ большего рода (т.е. рода $g \geqslant 2$ ) имеет состояния равновесия и, следовательно, не является минимальным. Аналогом минимального потока на такой поверхности является транзитивный поток, любая одномерная траектория которого всюду плотна на поверхности. Следуя [2], класс таких потоков без точек покоя нулевого индекса (т.е. без так называемых фальшивых седел или проходимых крошек) обозначим через $T_{g}$. Поток,

Работа вьполнена при поддержке Российского фонда фундаменталњных исследований (грант № 96-01-00236). 
любая одномерная траектория которого всюду плотна на поверхности, К. Гардинер [3] предложил называть сверхтранзитивныцм.

Для потоков класса $T_{g}$ на ориентируемой замкнутой поверхности $M$ полным топологическим инвариантом (с точностью до пересчета с помощью автоморфизма фундаментальной группы) является гомотопический класс врашения, введенный С.Х. Арансоном и В.З. Гринесом [2], который определяется для индивидуальной полутраектории, не имеющей точку покоя в качестве предельного множества. Поскольку в потоке класса $T_{g}$ гомотопический класс вращения одной полутраектории определяет гомотопический класс врашения любой другой полутраектории, то полный топологический инвариант такого потока можно представить в виде специального геодезического распределения (геодезического каркаса потока), состоящего из геодезических с теми же самыми асимптотическими направлениями, что и траектории потока. Такое представление полного топологического инварианта более удобно, поскольку множество геодезических каркасов наделяется естественной структурой топологического пространства, и можно рассмотреть задачу о зависимости геодезического каркаса от возмущения потока (или от параметра семейства потоков). Эту задачу можно рассмотреть для любого потока с непустьм геодезическим каркасом (т.е. имеюшего траектории, которые при поднятии на универсальную накрывающую уходят в бесконечность в определенном направлении).

Аналогичная задача о зависимости числа врашения Пуанкаре от возмушения потока на торе решалась многими исследователями. Зависимость геодезического каркаса от возмущения потока до настоящего времени не рассматривалась.

\section{§1. Основные определения и результаты}

Модель Пуанкаре плоскости Лобачевского. Пусть $\Delta$ - плоскость Лобачевского в виде модели Пуанкаре, т.е. $\Delta$ - единичный круг на комплексной $z$-плоскости, наделенный метрикой постоянной отрицательной кривизны, которая задается квадратичной формой $d s=2|d z| /\left(1-|z|^{2}\right)$. Окружность $S_{\infty}=\partial \Delta=(|z|=1)$ называется абсолютом. Согласно теореме об униформизации для любой ориентируемой замкнутой поверхности $M_{g}$ рода $g \geqslant 2$ найдется фуксова группа $Г$ сохраняющих ориентацию изометрий плоскости $\Delta$ такая, что $\Delta / \Gamma \cong M_{g}$. Обозначим через $\pi: \Delta \rightarrow \Delta / \Gamma \cong M_{g}$ естественную проекцию, которая является универсальньм накрьваюшим отображением.

Точка $\sigma \in S_{\infty}$ называется рациональной, если она является неподвижной точкой некоторого преобразования (отличного от тождественного) группы Г. Точки абсолюта, не являюшиеся рациональными, называются иррациональными.

Напомним, что геодезическим распределением на $M$ называется непустое замкнутое множество, состоящее из попарно непересекающихся геодезических, каждая из которых не имеет самопересечений. ${ }^{1}$ Геодезическое распределение называ-

\footnotetext{
${ }^{1}$ Иногда встречается термин "геодезическая ламинация". "Ламинация" - это слоение без особенностей на замкнутом множестве (подробнее см. [4]). На поверхности понятие ламинации, состоящей из геодезических (она и назьвается геодезической ламинацией), оказывается эквивалентньм понятию геодезического распределения, хотя формально в определении последнего накладываются менее ограничительные условия.
} 
ется минимальным, если оно не содержит собственных геодезических распределений.

Геодезический каркас потока. Наиболее общие условия существования асимптотического направления для полутраекторий накрывающего потока получил Д.В. Аносов [5]. Это позволяет построить геодезический каркас для потоков со стягиваемым множеством точек покоя.

Пусть $f^{t}$ - поток на ориентируемой замкнутой поверхности $M_{g}$ рода $g \geqslant 2$. Для $f^{t}$ имеется накрывающий поток $\bar{f}^{t}$ на $\Delta$ в силу проекции $\pi$. Для простоты изложения ограничимся рассмотрением потоков, точки покоя которых являются топологическими седлами. Рассмотрим траекторию $\bar{l}$ потока $\bar{f}^{t}$, которая в обоих направлениях стремится к разным точкам абсолюта $\alpha(\bar{l}) \neq \omega(\bar{l})$, где $\alpha(\omega)(\bar{l})$ означает $\alpha(\omega)$-предельное множество траектории $\bar{l}$. Обозначим через $\bar{L}(\bar{l})$ геодезическую, соединяющую точки $\alpha(\bar{l}), \omega(\bar{l})$ и ориентированную от $\alpha(\bar{l})$ к $\omega(\bar{l})$. Эта геодезическая $\bar{L}(\bar{l})$ называется соответствующей траектории $\bar{l}$. Аналогичное построение соответствующей геодезической проводится для обобщенной траектории потока $\bar{f}^{t}$ (т.е. объединения сепаратрисы и ее односторонних продолжений по Бендиксону) [6]-[8]. Отметим, что обобщенная траектория может состоять из счетного семейства сепаратрис и точек покоя потока $\bar{f}^{t}$. В этом случае обобщенная траектория является накрывающей для одностороннего негомотопного нулю контура потока $f^{t}$.

Обозначим через $\bar{G}\left(\bar{f}^{t}\right)$ семейство ориентированных геодезических, соответствующих всем траекториям и обобщенным траекториям потока $\bar{f}^{t}$. Тогда $\pi\left(\bar{G}\left(\bar{f}^{t}\right)\right) \stackrel{\text { def }}{=} G\left(f^{t}\right)$ является геодезическим распределением, которое назьвается геодезическим каркасом потока $f^{t}$.

Для транзитивных потоков и потоков типа Данжуа имеется эквивалентное определение геодезического каркаса (которое можно дать даже для некомпактной поверхности). Дадим вначале определение геодезического каркаса для квазиминимального множества потока. Напомним, что положительная (отрицательная) полутраектория незамкнутой траектории называется нетривиально рекуррентной в положительном (отрицательном) направлении, если эта полутраектория лежит в своем $\omega(\alpha)$-предельном множестве. Незамкнутая траектория называется нетривиально рекуррентной, если она нетривиально рекуррентна в обоих направлениях. Замькание (в теоретико-множественном смысле) нетривиально рекуррентной полутраектории называется көазиминимальным множеством.

Возьмем одну из накрывающих $\bar{l}$ для нетривиально рекуррентной траектории $l$, принадлежашей квазиминимальному множеству $Q$ потока $f^{t}$. В [2] доказано, что $\bar{l}$ в обоих направлениях стремится к разным точкам абсолюта. Поэтому для $\bar{l}$ существует соответствующая геодезическая $\bar{L}(\bar{l})$. Тогда $\pi(\bar{L}(\bar{l}))=L(l)$ является нетривиально рекуррентной геодезической без самопересечения на поверхности $M$. Ее топологическое замыкание $\operatorname{clos}[L(l)]$ является геодезическим распределением, которое не зависит от выбора нетривиально рекуррентной траектории из квазиминимального множества, поскольку в силу теоремы А.Г. Майера [9] любая нетривиально рекуррентная траектория из квазиминимального множества $Q$ всюду плотна в $Q$. Геодезическое распределение $\cos [L(l)]$ называется геодезическим каркасом квазиминимального множества $Q$ и обозначается через $G(Q)$. 
Для транзитивного потока $f^{t}$ квазиминимальное множество $Q$ совпадает со всей поверхностью $M$, и геодезический каркас $G(M)$ является геодезическим каркасом $G\left(f^{t}\right)$ потока $f^{t}$.

Геодезический каркас называется рациональным, если он не содержит нетривиальных рекуррентных геодезических. Геодезический каркас назьвается иррациональным, если он состоит из нетривиальных рекуррентных геодезических. Любая накрывающая из рационального (соответственно иррационального) каркаса является геодезической с рациональњыми (соответственно иррациональньми) концевыми точками. Отметим, что в силу замкнутости геодезического распределения любой рациональный геодезический каркас содержит замкнутые геодезические.

Геодезический каркас назьвается неприводимым, если любая негомотопная нулю замкнутая кривая пересекает, по крайней мере, одну геодезическую из геодезического каркаса. Неприводимость геодезического каркаса означает, что любая компонента связности дополнения к этому каркасу односвязна.

Обозначим через $\chi^{1}(M)$ пространство $C^{1}$-векторных полей на $M=M_{g}$, снабженное $C^{1}$-топологией, и через $\widetilde{\chi}^{1}(M) \subset \chi^{1}(M)$ - векторные поля, состоянием равновесия которых являются только грубые (гиперболические) седла. Обозначим через $S_{g}^{t}$ множество $C^{1}$-потоков на $M$, индуцируемых векторными полями $\tilde{\chi}^{1}(M)$, и через $H S_{g}^{t} \subset S_{g}^{t}$ - подмножество потоков, принадлежащих классу $T_{g}$. Нетрудно видеть, что множество $H S_{g}^{t}$ состоит из сверхтранзитивных потоков.

Так как каждый поток $f^{t} \in S_{g}^{t}$ не имеет точек покоя положительного индекса, то $G\left(f^{t}\right) \neq \varnothing$. Из теоремы А.Г. Майера [9] вытекает, что геодезический каркас $G\left(f^{t}\right)$ потока класса $T_{g}$ иррациональный и является минимальньм геодезическим распределением. Из сверхтранзитивности потока $f^{t}$ вытекает неприводимость геодезического каркаса $G\left(f^{t}\right)$.

Полньй прообраз $\pi^{-1}\left(G\left(f^{t}\right)\right)$ геодезического каркаса $G\left(f^{t}\right)$ потока $f^{t}$ является геодезическим распределением на $\Delta$. Если поток $f^{t}$ транзитивный, то дополнение к $\pi^{-1}\left(G\left(f^{t}\right)\right)$ состоит из открытых гиперболических $4 n$-многоугольников $(n \geqslant 1)$, вершины которых лежат на абсолюте. Если дополнение к $\pi^{-1}\left(G\left(f^{t}\right)\right)$ содержит только четырехугольники, то каркас $G\left(f^{t}\right)$ будем называть простейшим. Ясно, что поток $f^{t} \in H S_{g}^{t}$ имеет простейший геодезический каркас.

Топология на множестве геодезических. Обозначим через $T_{1}(M)$ единичное касательное расслоение над поверхностью $M=M_{g}$ и пусть $p: T_{1}(M) \rightarrow M-$ естественная проекция, отображающая единичный касательный вектор в точку его касания. На 3 -мерном многообразии $T_{1}(M)$ определен геодезический поток $G^{t}$.

Введем на множестве $\mathrm{Geo}(M)$ ориентированных геодезических поверхности $M$ структуру топологического пространства. Для этого достаточно ввести топологию на множестве $\operatorname{Tr}\left(G^{t}\right)$ траекторий геодезического потока $G^{t}$. Пусть $\tilde{l}$ - траектория потока $G^{t}$. Возьмем компактную дугу $S$ траектории $\tilde{l}$ и трубчатую окрестность $T(S)$ дуги $S$, состоящую из дуг траекторий потока $G^{t}$. Обозначим через $\widetilde{T}(S)$ множество траекторий потока $G^{t}$, проходящих через $T(S)$. Семейство множеств $\widetilde{T}(S)$ для всевозможных трубчатых окрестностей $T(S)$ и дуг $S \subset \tilde{l}$ образует предбазу семейства окрестностей траектории $\tilde{l}$.

Пусть $G l_{1}, G l_{2}$ - геодезические распределения на поверхности $M$. Будем говорить, что $G l_{1}$ лежит в $U$-окрестности $G l_{2}$, если $p^{-1}\left(G l_{1}\right)$ принадлежит $U$-окрест- 
ности $p^{-1}\left(G l_{2}\right)$.

Основные результаты статьи. Следующая теорема означает, что неприводимьй простейший иррациональный геодезический каркас сверхтранзитивного потока непрерьвно зависит от возмущения потока (что аналогично непрерывной зависимости иррационального числа врашения Пуанкаре от возмушения потока).

ТЕОРЕМа 3.1. Пусть $f^{t}$ - сверхтранзитивный поток на замкнутой ориентируемой поверхности $M$ рода $g \geqslant 2$, индуцируемый векторным полем $v \in \tilde{\chi}^{1}(M)$, и пусть $U$ - окрестность геодезического каркаса $G\left(f^{t}\right)$. Тогда существует окрестность $O^{1}(v) \subset \chi^{1}(M)$ векторного поля $v$ такая, что любое векторное поле $w \in O^{1}(v)$ индуцирует поток $g^{t}$ с непустым геодезическим каркасом $G\left(g^{t}\right)$, который принадлежит окрестности $U$.

СЛЕДСТВИЕ 3.1. Пусть выполняются условия теоремы 3.1. Тогда для любого конечного набора геодезических $L_{1}, \ldots, L_{k} \in G\left(f^{t}\right)$ и любых их окрестностей $U\left(L_{1}\right), \ldots, U\left(L_{k}\right)$ существует окрестность $O^{1}(v) \subset \chi^{1}(M)$ векторного поля $v$ такая, что любое векторное поле $w \in O^{1}(v)$ индуцирует поток $g^{t} c$ непустым геодезическим каркасом $G\left(g^{t}\right)$, который принадлежст пересечению $U\left(L_{1}\right) \cap \cdots \cap U\left(L_{k}\right)$.

ЗАмечАниЕ. Теорема 3.1, вообше говоря, становится неверной, если не требовать гиперболичности седел. Можно построить пример сверхтранзитивного потока $f^{t}$, имеющего седло с восемњю сепаратрисами, и возмушение потока $f^{t}$, при котором геодезический каркас $G\left(f^{t}\right)$ не меняется непрерьвно. Идея примера состоит в том, что дополнение к $G\left(f^{t}\right)$ содержит идеальный гиперболический восьмиугольник, который при соответствующем возмущении потока $f t$ "разваливается" на два четырехугольника (седло с восемњю сепаратрисами при возмушении потока "разваливается" на два седла с четырьмя сепаратрисами каждый, и при этом не образуются сепаратрисные связи). Возникающая "диагональ" восьмиугольника не лежит в некоторой окрестности геодезического каркаса $G\left(f^{t}\right)$.

Аналогичные соображения показывают, что в теореме 3.1 нельзя условие сверхтранзитивности потока $f^{t}$ заменить на транзитивность, поскольку наличие сепаратрисной связи между двумя гиперболическими седлами у транзитивного потока вновь приводит к существованию гиперболического восьмиугольника в дополнении к $G\left(f^{t}\right)$.

Следующая теорема означает, что неприводимьй иррациональньй геодезический каркас “неустойчив", т.е. существуют сколь угодно малые возмущения потока, которые разрушают такой каркас (однако, в силу теоремы 3.1 "разрушение" каркаса происходит непрерьвно).

ТЕОРема 3.2. Пусть $f^{t}$ - сверхтранзитивный поток на замкнутой ориентируемой поверхности $M$ рода $g \geqslant 2$, индуиируемый векторным полем $v \in \widetilde{\chi}^{1}(M)$. Тогда для любой окрестности $U$ геодезического каркаса $G\left(f^{t}\right)$ и любой окрестности $O^{1}(v) \subset \chi^{1}(M)$ существует векторное поле $w \in O^{1}(v)$ такое, что $y$ потока $g^{t}$, индуцированного полем $w$, геодезический каркас $G\left(g^{t}\right)$ рациональный и принадлежст окрестности $U$. 
Что касается рационального каркаса, то нетрудно найти примеры как непрерывной зависимости, так и не непрерьвной зависимости каркаса от потока. Пример непрерывной зависимости дает поток Морса-Смейла (которьй, очевидно, имеет рациональный геодезический каркас). Так как такой поток структурно устойчив, то при малых возмущениях потока его геодезический каркас не меняется. Следующие две теоремы описывают возможный сценарий разрушения рационального каркаса.

ТЕОРема 4.1. На замкнутой ориентируемой поверхности $M$ рода $g \geqslant 2$ существует однопараметрическое семейство $C^{\infty}{ }_{-}$потоков $f_{\mu}^{t}$, непрерьвно зависящих от параметра $\mu \in[0,1]$, такое, что выполняются следующие утверждения.

1. Для всех $\mu \in[0,1)$ поток $f_{\mu}^{t}$ имеет неприводимый иррациональный каркас $G\left(f_{\mu}^{t}\right) \neq \varnothing$, не зависящий от параметра $\mu$.

2. Поток $f_{1}^{t}$ имеет рациональньий каркас $G\left(f_{1}^{t}\right)$.

3. Существует окрестность $U$ каркаса $G\left(f_{1}^{t}\right)$ такая, что $G\left(f_{\mu}^{t}\right) \notin U$ при $\mu \in[0,1)$.

ТЕОРема 4.2. На замкнутой ориентируемой поверхности $M$ рода $g \geqslant 2$ существует однопараметрическое семейство $C^{\infty}$-потоков $f_{\mu}^{t}$, непрерывно зависящих от параметра $\mu \in[0,1]$, такое, что выполняются следующие утверждения.

1. Для всех $\mu \in[0,1]$ поток $f_{\mu}^{t}$ имеет рациональный каркас $G\left(f_{\mu}^{t}\right) \neq \varnothing$, не зависящий от параметра $\mu \in[0,1)$.

2. Существует окрестность $U$ каркаса $G\left(f_{1}^{t}\right)$ такая, что $G\left(f_{\mu}^{t}\right) \notin U$ при $\mu \in[0,1)$.

Разрывность рационального геодезического каркаса в общем случае не удивительна, поскольку на торе существуют потоки с рациональньп числом вращения, которое меняется скачкообразно при произвольно малых возмущениях этих потоков. При этом разрывность рационального числа вращения связана с исчезновением глобальной секущей потока [10], [11] (напомним, что число вращения Пуанкаре гомеоморфизмов окружности меняется непрерьвно, поэтому если исходньй поток и близкие ему потоки на торе имеют глобальную секущую, то число вращения исходного потока также меняется непрерывно).

Сформулируем теперь теорему о существовании одной бифуркации геодезических каркасов (которая соответствует некоторому семейству потоков).

Теорема 5.1. На замкнутой ориентируемой поверхности $M$ рода $g \geqslant 2$ существует однопараметрическое семейство $C^{\infty}$-потоков $f_{\mu}^{t}$, непрерьвно зависящих от параметра $\mu \in[0,1)$, такое, что выполняются следующие утверждения.

1. Для всех $\mu \in[0,1)$ поток $f_{\mu}^{t}$ имеет рачиональный каркас $G\left(f_{\mu}^{t}\right) \neq \varnothing$.

2. Длины замкнутых геодезических в $G\left(f_{\mu}^{t}\right)$ равномерно стремятся к бесконечности при $\mu \rightarrow 1$. 
ЗАмечАнИЕ. В теореме 5.1 можно потребовать, чтобы рациональный каркас $G\left(f_{\mu}^{t}\right)$ состоял только из замкнутых геодезических, и точки покоя потоков $f_{\mu}^{t}$ были гиперболическими седлами и узлами, расположенными в фиксированных точках поверхности.

Непосредственно из доказательства теоремы 5.1 следует, что семейство потоков $f_{\mu}^{t}, \mu \in[0,1)$, можно продолжить до потока $f_{1}^{t}$ таким образом, чтобы геодезический каркас $G\left(f_{1}^{t}\right)$ был либо рациональным, либо иррациональньм, либо пустым. В последнем случае мы будем говорить, что имеет место коллапс геодезического каркаса.

Следующая теорема описывает некоторые динамические свойства потока, на котором происходит коллапс геодезического каркаса.

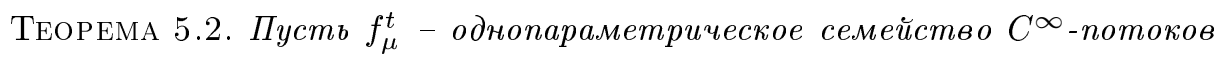
на замкнутой ориентируемой поверхности $M$ рода $g \geqslant 2$, непрерывно зависящих от параметра $\mu \in[0,1]$. Предположим, что

1) для всех $\mu \in[0,1)$ поток $f_{\mu}^{t}$ имеет рациональный каркас $G\left(f_{\mu}^{t}\right) \neq \varnothing$;

2) длины замкнутых геодезических в $G\left(f_{\mu}^{t}\right)$ равномерно стремятся к бесконечности при $\mu \rightarrow 1$;

3) точки покоя потоков $f_{\mu}^{t}$ при $\mu \in[0,1)$ расположены в фиксированных точках поверхности и их конечное число.

Тогда если $G\left(f_{1}^{t}\right)=\varnothing$, то поток $f_{1}^{t}$ имеет бесконечно много точек покоя.

Статья организована следующим образом. Во втором параграфе мы доказываем ряд необходимых для дальнейшего результатов. В частности, доказывается следуюшая теорема об "устойчивости” глобальной секущей, которая имеет самостоятельный интерес.

ТЕОРема 2.1. Пусть поток $f^{t}$ на $M^{n}$, индуцируемый векторным полем $v \in \chi^{1}\left(M^{n}\right)$, имеет глобальную секущую $C$. Предположим, что точки покоя потока $f^{t}$ являются грубими. Тогда существует окрестность $O^{1}(v) \subset$ $\chi^{1}\left(M^{n}\right)$ поля $v$ такая, что любое векторное поле $w \in O^{1}(v)$ индуцирует поток $g^{t}$, который имеет глобальную секущую $C$.

В третьем параграфе доказываются теоремы 3.1 и 3.2 , в четвертом-теоремы 4.1 и 4.2, в пятом - теоремы 5.1 и 5.2 .

Мы благодарим В.З. Гринеса, М.И. Малкина и И. Николаева за полезные обсуждения.

\section{§2. Вспомогательные результаты}

Нам понадобится теорема об "устойчивости" глобальной секушей относительно возмушения потока. Эта теорема имеет самостоятельный интерес для теории бифуркаций, и мы ее докажем для потока на $n$-мерном компактном многообразии.

Напомним, что глобальной секущей потока $f^{t}$ на $n$-мерном компактном многообразии $M^{n}$ называется такое вложенное в $M^{n}$ компактное подмногообразие $C$ коразмерности один, что любая одномерная траектория потока $f^{t}$ пересекает трансверсально $C$ при $t \rightarrow-\infty$ или $t \rightarrow+\infty$. 
ТЕОРема 2.1. Пусть поток $f^{t}$ на $M^{n}$, индуцируемый векторным полем $v \in \chi^{1}\left(M^{n}\right)$, имеет глобальную секущую $C$. Предположим, что точки покоя потока $f^{t}$ являются грубыми. Тогда существует окрестность $O^{1}(v) \subset$ $\chi^{1}\left(M^{n}\right)$ поля $v$ такая, что любое векторное поле $w \in O^{1}(v)$ индуцирует поток $g^{t}$, который имеет глобальную секущую $C$.

ДокАЗАТЕЛЬСтво. В силу компактности подмногообразия $C$ сушествует окрестность $O_{1}^{1}(v) \subset \chi^{1}\left(M^{n}\right)$ поля $v$ такая, что $C$ трансверсальна любому векторному полю $w \in O_{1}^{1}(v)$.

Покажем сначала, что существуют число $\delta>0$ и окрестность $O_{2}^{1}(v)$ поля $v$ такие, что любая одномерная траектория потока $g^{t}$, индуцируемого векторньм полем из $O_{2}^{1}(v)$, покидает $\delta$-окрестность множества точек покоя $\operatorname{Fix}\left(f^{t}\right)$ при $t \rightarrow-\infty$ или $t \rightarrow+\infty$.

Число точек покоя потока $f^{t}$ конечно, поэтому достаточно доказать следующее

УТВЕРЖДЕНИЕ. Пусть на п-мерном евклидовом пространстве $\mathbb{R}^{n}$ задан поток $F_{0}^{t}$, индуиируемый векторным полем $\vec{V}$ класса $C^{1}$, который в начале декартовых координат имеет грубое состояние равновесия О. Тогда существует окрестность $U_{0}$ точки $O$ и окрестность $O^{1}(\vec{V}) \subset \chi^{1}\left(\mathbb{R}^{n}\right)$ поля $\vec{V}$ такие, что у любого потока $F^{t}$, индуцируемого векторным полем $\vec{W} \in O^{1}(\vec{V})$, нет траекторий, кроме точки покоя $O$, целиком лежсащих в $U_{0}$.

Действительно, пусть поток $F_{0}^{t}$ задается уравнением

$$
\dot{\mathbf{x}}=A \mathbf{x}+o(\mathbf{x})
$$

где $A \mathbf{x}$ - линейная часть уравнения. У близких потоков с помощью линейной замены переменных состояние равновесия можно переместить в точку $O$. Поэтому не нарушая общности будем считать, что у всех близких потоков состояние равновесия имеет координаты $\mathbf{x}=0$.

Если матрица $A$ в уравнении (1) имеет простые собственные числа, то в силу грубости $O$ с помощью линейной замены уравнение (1) можно преобразовать к виду:

$$
\dot{x}_{k}=q_{k} x_{k}+o\left(\mathbf{x}^{2}\right), \quad \dot{x}_{j}=a_{j} x_{j}-b_{j} x_{j+1}+o\left(\mathbf{x}^{2}\right), \quad \dot{x}_{j+1}=b_{j} x_{j}+a_{j} x_{j+1}+o\left(\mathbf{x}^{2}\right),
$$

где $q_{k}$ - действительные собственные числа, $q_{j}=a_{j}+i b_{j}$ - комплексные собственные числа. Проведем доказательство требуемого утверждения для случая простых собственных чисел. В случае же, когда характеристические числа $q_{k}$ являются кратными корнями характеристического уравнения, то рассуждения аналогичны, и мы их опустим.

Рассмотрим функцию

$$
f(\mathbf{x})=x_{1}^{2}+x_{2}^{2}+\cdots+x_{n}^{2}
$$

и функцию

$$
h(\mathbf{x})=\frac{1}{4}(\overrightarrow{\operatorname{grad}(f)}, \vec{V}),
$$


равную четверти скалярного произведения векторных полей $\overrightarrow{\operatorname{grad}(f)}, \vec{V}$. Найдем производную по времени от функции $h(\mathbf{x}(t))$, где $\mathbf{x}=\mathbf{x}(t)$ - уравнение траектории потока $F_{0}^{t}$. В силу (2) имеем

$$
\frac{d}{d t} h(\mathbf{x}(t))=\cdots+x_{k}^{2} q_{k}^{2}+\cdots+a_{j}^{2} x_{j}^{2}+a_{j}^{2} x_{j+1}^{2}+\cdots+o\left(\mathbf{x}^{3}\right) .
$$

Следовательно, в некоторой компактной области $D_{0}$, содержащей точку $O$, будет вьполнено неравенство $\frac{d}{d t} h(\mathbf{x}(t)) \geqslant \alpha_{0}>0$, если $\mathbf{x}(t) \subset D_{0}$. Отсюда вытекает существование окрестности $U_{0}$ точки $O$ и окрестности $O^{1}(\vec{V}) \subset \chi^{1}\left(\mathbb{R}^{n}\right)$ поля $\vec{V}$ таких, что для любого потока $F^{t}$, индуцируемого векторным полем $\vec{W} \in O^{1}(\vec{V})$, и любой его траектории $\widetilde{\mathbf{x}}(t)$ выполняется неравенство $\frac{d}{d t} h(\widetilde{\mathbf{x}}(t))>0$, если $\widetilde{\mathbf{x}}(t) \subset U_{0}$. Покажем, что окрестности $U_{0}, O^{1}(\vec{V})$ являются искомыми. Предположим противное. Тогда сушествует последовательность векторных полей $\vec{W}_{i} \in O^{1}(\vec{V})$, сходящихся к $\vec{V}$, у которых имеется хотя бы по одной траектории $\widetilde{\mathbf{x}}_{i}(t)$, целиком лежащих в $U_{0}$. Для каждой такой траектории найдется $(n-1)$-мерная сфера

$$
S_{i}: x_{1}^{2}+x_{2}^{2}+\cdots+x_{n}^{2}-r_{i}^{2}=0
$$

такая, что траектория $\widetilde{\mathbf{x}}_{i}(t)$ касается $S_{i}$ и принадлежит замкнутой области $g_{i}$, ограниченной сферой $S_{i}$. Отсюда следует, что в точке касания $m_{i}$ траектории $\widetilde{\mathbf{x}}_{i}(t)$ со сферой $S_{i}$ будет выполняться равенство

$$
\left(\overrightarrow{\operatorname{grad}(f)}, \vec{W}_{i}\right)=0
$$

Так как траектория $\widetilde{\mathbf{x}}_{i}(t)$ касается $S_{i}$ и принадлежит области, ограниченной сферой $S_{i}$, то функция

$$
h\left(\widetilde{\mathbf{x}}_{i}(t)\right)=\frac{1}{4}\left(\overrightarrow{\operatorname{grad}(f)}, \vec{W}_{i}\right)
$$

в малой окрестности $m_{i}$ при возрастании времени $t$ является невозрастающей функцией (обращающейся в нуль в точке $m_{i}$ ). Следовательно, производная по направлению вдоль $\widetilde{\mathbf{x}}_{i}(t)$ от функции $h\left(\widetilde{\mathbf{x}}_{i}(t)\right)$ в точке $m_{i}$ будет меньше или равна нулю, что противоречит неравенству $\frac{d}{d t} h(\widetilde{\mathbf{x}}(t))>0$.

Таким образом, сушествуют число $\delta>0$ и окрестность $O_{2}^{1}(v)$ поля $v$ такие, что любая одномерная траектория потока $g^{t}$, индуцируемого векторным полем из $O_{2}^{1}(v)$, покидает $\delta$-окрестность множества точек покоя $\operatorname{Fix}\left(f^{t}\right)$ при $t \rightarrow-\infty$ или $t \rightarrow+\infty$.

Предположим, что теорема не верна. Тогда существуют последовательности векторных полей $w_{k} \in O_{1}^{1}(v) \cap O_{2}^{1}(v)$ и траекторий $l_{k}$ потоков, индуцируемых полями $w_{k}$, такие, что векторные поля $w_{k}$ сходятся к $v$ при $k \rightarrow \infty$ и траектории $l_{k}$ не пересекают $C$. Так как $l_{k}$ покидают $\delta$-окрестность $U_{\delta}$ множества $\operatorname{Fix}\left(f^{t}\right)$, то существуют точки $x_{k} \in l_{k}$, не принадлежашие $U_{\delta}$. Не уменьшая обшности, можно считать, что последовательность $x_{k}$ сходится к некоторой точке $x_{0} \in M^{n}$. Так как $x_{k} \notin U_{\delta}$, то $x_{0}$ не является точкой покоя потока $f^{t}$. Следовательно, траектория $l_{0}$ потока $f^{t}$, проходящая через точку $x_{0}$, пересекает $C$. По теореме о непрерывной зависимости дуг траекторий от начальных условий траектории $l_{k}$ также должны пересекать $C$ при достаточно большом $k$. Полученное противоречие доказывает теорему. 
СлеДСТвИЕ 2.1. Предположим, что поток $f^{t} \in S_{g}^{t}$ на $M$, индуцируемый векторныцм полем $v \in \widetilde{\chi}^{1}(M)$, имеет глобальную секущую $C$. Тогда существует окрестность $O^{1}(v) \subset \chi^{1}\left(M^{n}\right)$ такая, что любое векторное поле $w \in O^{1}(v)$ индуцирует поток $g^{t}$, который имеет глобальную секущую $C$.

Будем говорить, что две направленные геодезические $L_{1}, L_{2}$ на плоскости Лобачевского $\Delta \varepsilon$-близки, если их начальные и конечные точки соответственно $\varepsilon$-близки на абсолюте в евклидовой метрике на $S_{\infty}$.

Следуюшие две леммы нам понадобятся при доказательстве теоремы 3.1.

Лемма 2.1. Пусть $L \subset M$ - ориентированная геодезическая, и пусть $V(L)$ - ее окрестность в $\mathrm{Geo}(M)$. Пусть $\bar{L} \subset \Delta$ - накривающая для $L$ геодезическая. Тогда существует $\delta>0$ такое, что если геодезическая $\bar{L}_{1}$ $\delta$-близка $к \bar{L}$ в евклидовом смицсле, то $\pi\left(\bar{L}_{1}\right) \subset V(L)$.

ДокАЗАТЕЛЬСтво. В силу определения топологической структуры на множестве $\mathrm{Geo}(M)$ для данной окрестности $V(L)$ сушествует геодезический сегмент $\Sigma$ такой, что $\Sigma$ перпендикулярен к $L$ в некоторой точке, и если другая геодезическая $L_{1}$ пересекает $\Sigma$ под углом, близким к нормальному, то $L_{1} \subset V(L)$. Теперь утверждение леммы следует из геометрических соображений, поскольку геодезическими на $\Delta$ являются дуги евклидовых окружностей, перпендикулярных абсолюту.

ЛЕмма 2.2. Пусть выполняются условия теоремъ 3.1 , и пусть $C_{0}-$ простая замкнутая геодезическая, которая пересекается со всеми геодезическими из геодезического каркаса $G\left(f^{t}\right)$. Пусть $\bar{I}$ - конечный геодезический сегмент на $\Delta$, который накрывает $C_{0}$. Обозначим через $\bar{G}\left(\bar{f}^{t}\right)=\pi^{-1}\left(G\left(f^{t}\right)\right)$ полный прообраз геодезического каркаса $G\left(f^{t}\right)$. Тогда для любой окрестности $U$ каркаса $G\left(f^{t}\right)$ существует число $\delta>0$ такое, что если геодезическая $\bar{L}_{1} \subset \Delta$-бллизка в евклидовом смисле $к$ некоторой геодезической $\bar{L} \in \bar{G}\left(\bar{f}^{t}\right)$, $\bar{L} \cap \bar{I} \neq \varnothing$, mo $\pi\left(\bar{L}_{1}\right) \in V$.

ДоКАЗАТЕльство. В силу леммы 2.1 для любой геодезической $\bar{L} \in \bar{G}\left(\bar{f}^{t}\right)$, $\bar{L} \cap \bar{I} \neq \varnothing$, сушествует число $\delta(\bar{L})>0$ такое, что если $\bar{L}_{1} \delta(\bar{L})$-близка к $\bar{L}$ в евклидовом смысле, то $\pi\left(\bar{L}_{1}\right) \in U$. Пусть $\delta_{\text {max }}(\bar{L})$ есть супремум всех таких $\delta(\bar{L})$. Из замкнутости геодезического каркаса $\bar{G}\left(\bar{f}^{t}\right)$ и из конечности (и, следовательно, компактности) геодезического сегмента $\bar{I}$ следует, что

$$
\min _{\bar{L} \in \bar{G}\left(\bar{f}^{t}\right), \bar{L} \cap \bar{I} \neq \varnothing} \delta_{\max }(\bar{L})=\alpha>0 .
$$

Тогда мы можем взять $\delta=\alpha / 2$.

\section{§3. Непрерывность иррационального каркаса}

В этом параграфе мы докажем две теоремы 3.1 и 3.2 и следствие 3.1 (формулировки см. в введении).

ДОКАЗАТЕЛЬСТВО ТЕОРЕМЫ 3.1 разобъем на ряд шагов.

Шаг 1. Поток $f^{t} \in H T S_{g}^{t}$ имеет глобальную секущую $C$. 
Поскольку $f^{t}$ имеет нетривиально рекуррентные траектории, то существует негомотопная нулю простая замкнутая трансверсаль $C$ потока $f^{t}[6],[12]$. Так как $f^{t}$ - сверхтранзитивный поток, то $C$ - глобальная секущая.

Шаг 2. Существует простая замкнутая геодезическая $C_{0}$, гомотопная $C$, такая, что все геодезические из геодезического каркаса $G\left(f^{t}\right)$ пересекают $C_{0}$ трансверсально.

Так как $C$ негомотопна нулю, то сушествует свободно гомотопная $C$ замкнутая геодезическая $C_{0}$. Кривая $C$ простая, поэтому $C_{0}$ также простая. Возьмем произвольную геодезическую $L$ из $G\left(f^{t}\right)$ и пусть $\bar{L}$ - ее поднятие на $\Delta$. По определению геодезического каркаса, сушествует траектория или обобшенная траектория $\bar{l}$, для которой $\bar{L}$ является соответствующей, т.е. $\bar{L}$ и $\bar{l}$ имеют общие концевые точки на абсолюте. Так как $C$ - глобальная секущая, то существует поднятие $\bar{C}$ кривой $C$ такое, что $\bar{l} \cap \bar{C} \neq \varnothing$. В силу гомотопности $C$ и $C_{0}$, сушествует поднятие $\bar{C}_{0}$ кривой $C_{0}$ такое, что $\bar{C}$ и $\bar{C}_{0}$ имеют общие конщевые точки на абсолюте. Но тогда из $\bar{l} \cap \bar{C} \neq \varnothing$ вытекает, что $\bar{L} \cap \bar{C}_{0} \neq \varnothing$, откуда следует требуемое утверждение.

Шаг 3. (Воспользуемся обозначениями доказательства шага 2.) Пусть $\bar{I}$ конечная дуга на кривой $\bar{C}$, которая имеет конгруәнтные концевые точки $\bar{a}, \bar{b} \in \bar{C}$ и которая, следовательно, накрывает $C$. Не уменьиая общности, можно считать, что через точки $\bar{a}, \bar{b}$ дуги $\bar{I}$ проходят траектории $\bar{l}(\bar{a}), \bar{l}(\bar{b})$ накрывающего потока, не являющиеся сепаратрисами. Пусть $\bar{g}(\bar{l}(\bar{a})), \bar{g}(\bar{l}(\bar{b}))$ - соответствующие геодезические траекторий $\bar{l}(\bar{a}), \bar{l}(\bar{b})$. Тогда геодезические $\bar{g}(\bar{l}(a)), \bar{g}(\bar{l}(b))$ конгруэнтны и пересекают геодезическую $\bar{C}_{0}$ в конгруэнтных moчкаx.

Так как точки $\bar{a}, \bar{b}$ конгруэнтны, то существует преобразование $\gamma \in \Gamma$ такое, что $\gamma(\bar{a})=\bar{b}$, и, следовательно, $\gamma(\bar{l}(\bar{a}))=\bar{l}(\bar{b})$ (поскольку $\gamma$ переводит траектории накрываюшего потока в траектории). Так как концевые точки траекторий $\bar{l}(\bar{a}), \bar{l}(\bar{b})$ и геодезических $\bar{g}(\bar{l}(\bar{a})), \bar{g}(\bar{l}(\bar{b}))$ соответственно совпадают, то $\gamma(\bar{g}(\bar{l}(\bar{a})))=\bar{g}(\bar{l}(\bar{b}))$, т.е. геодезические $\bar{g}(\bar{l}(\bar{a})), \bar{g}(\bar{l}(\bar{b}))$ конгруэнтны. Из того, что кривая $C$ простая, вытекает равенство $\gamma(\bar{C})=\bar{C}$, которое влечет равенство $\gamma\left(\bar{C}_{0}\right)=\bar{C}_{0}$. Так как траектории $\bar{l}(\bar{a}), \bar{l}(\bar{b})$ пересекают $\bar{C}$, геодезические $\bar{g}(\bar{l}(\bar{a})), \bar{g}(\bar{l}(\bar{b}))$ пересекают $\bar{C}_{0}$ в некоторых точках $\bar{a}_{0}, \bar{b}_{0}$ соответственно. В силу $\gamma(\bar{g}(\bar{l}(\bar{a})))=\bar{g}(\bar{l}(\bar{b})), \gamma\left(\bar{C}_{0}\right)=\bar{C}_{0}$ получаем $\gamma\left(\bar{a}_{0}\right)=\bar{b}_{0}$, т.е. точки $\bar{a}_{0}, \bar{b}_{0}$ конгруэнтны.

Шаг 4. Обозначим через $\bar{I}_{0}$ отрезок на геодезической $\bar{C}_{0}$ с концевыми точками $\bar{a}_{0}, \bar{b}_{0}$. Так как эти точки согласно шагу 3 конгруэнтны, то $\bar{I}_{0}$ накрывает $C_{0}$. Согласно лемме 2.2 для данной окрестности $U$ каркаса $G\left(f^{t}\right)$ сушествует число $\delta>0$ такое, что если геодезическая $\bar{L}_{1} \subset \Delta \delta$-близка в евклидовом смысле к некоторой геодезической $\bar{L} \in \bar{G}\left(\bar{f}^{t}\right), \bar{L} \cap \bar{I}_{0} \neq \varnothing$, то $\pi\left(\bar{L}_{1}\right) \in U$.

Шаг 5. Через произвольную точку $\bar{x} \in \bar{I}$ проходит либо траектория $\bar{l}(\bar{x})$ накрывающего потока $\bar{f}^{t}$, не являющаяся сепаратрисой, либо две обобщенные траектории $\bar{l}_{1}(\bar{x}), \bar{l}_{2}(\bar{x})$, кажсдая из которых является продолэсением по Бендиксону (с одной из двух сторон) сепаратрисъи $\bar{l}(\bar{x})$. Тогда в любом случае для траектории $\bar{l}(\bar{x})$ или для каждой обобщенной траектории $\bar{l}_{i}(\bar{x})$ существуют поднятия $\bar{C}_{1}(\bar{x}), \bar{C}_{2}(\bar{x})$ трансверсали $C$ такие, что концевые точки кривых $\bar{C}_{1}(\bar{x}), \bar{C}_{2}(\bar{x})$ ограничивают на абсолюте непересекающиеся дуги $\bar{d}_{1}(\bar{x}), \bar{d}_{2}(\bar{x})$, кажсдая из которых содержит по одной концевой точке либо 
траектории $\bar{l}(\bar{x})$, либо кривой $\bar{l}_{i}(\bar{x})$, и кажсдая из которых имеет евклидову длину меньиую, чем $\delta$ (см. рис. 1 ).

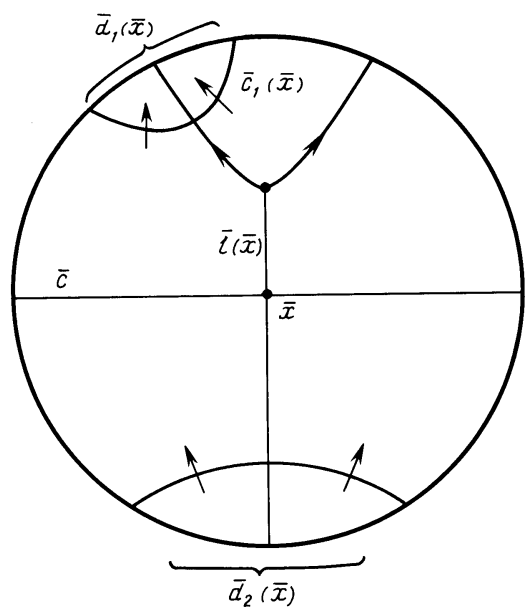

Рис. 1

Рассмотрим случай, когда через точку $\bar{x} \in \bar{I}$ проходит траектория $\bar{l}(\bar{x})$, не являющаяся сепаратрисой. Тогда $\bar{l}(\bar{x})$ является накрывающей для нетривиально рекуррентной траектории $l$ потока $f^{t}$. Так как $C$ - глобальная секущая, то $l$ пересекает $C$ в обоих направлениях в счетном множестве точек. Отсюда вытекает, что имеются две последовательности трансверсалей $\bar{C}_{n}, \bar{C}_{-n} \in \pi^{-1}(C), \bar{C}_{ \pm n} \cap \bar{l} \neq \varnothing$, таких, что $\bar{C}_{n}$ сходятся к $\omega$-предельному множеству $\omega(\bar{l})$ траектории $\bar{l}(\bar{x})$, а кривые $\bar{C}_{-n}$ сходятся к $\alpha$-предельному множеству $\alpha(\bar{l})$ траектории $\bar{l}(\bar{x})$. Оба множества $\omega(\bar{l}), \alpha(\bar{l})$ являются точками, лежащими на абсолюте [2]. Отсюда следует требуемое утверждение для случая, когда траектория $\bar{l}(\bar{x})$ не является сепаратрисой. В случае, когда через точку $\bar{x} \in \bar{I}$ проходит обобщенная траектория $\bar{l}_{i}(\bar{x})$, доказательство аналогично, поскольку $\bar{l}_{i}(\bar{x})$ состоит из двух накрьвающих для нетривиально рекуррентных полутраекторий.

Шаг 6. Согласно следствию 2.1 сушествует окрестность $O_{1}^{1}(v) \subset \chi^{1}(M)$ векторного поля $v$ такая, что любое векторное поле $w \in O_{1}^{1}(v)$ индуцирует поток $g^{t}$, которьй имеет глобальную секушую $C$. Так как состояниями равновесия поля $v$ являются грубые седла, то можно считать, что любое векторное поле $w \in O_{1}^{1}(v)$ имеет также состояниями равновесия только грубые седла. Другими словами, $g^{t} \in S_{g}^{t}$. Отсюда вытекает, что векторное поле $w \in O_{1}^{1}(v)$ индуцирует поток $g^{t}$ с непустым геодезическим каркасом $G\left(g^{t}\right)$.

Для траекторий потоков $\bar{g}^{t}$, накрывающих потоки $g^{t} \in S_{g}^{t}$, введем следующее обозначение. Пусть через точку $\bar{x} \in \Delta$ проходит траектория $\bar{l}^{\prime}$ потока $g^{t}$. Если $\bar{l}^{\prime}$ не сепаратриса, то положим $G E\left(\bar{x}, \bar{g}^{t}\right)=\bar{l}^{\prime}$. Если $\bar{l}^{\prime}-$ сепаратриса, то $G E\left(\bar{x}, \bar{g}^{t}\right)$ означает глобальное продолжение по Бендиксону сепаратрисы $\bar{l}^{\prime}$. Другими словами, $G E\left(\bar{x}, \bar{g}^{t}\right)$ означает объединение обобщенных траекторий потока $\bar{g}^{t}$, проходящих через точку $\bar{x}$. Отметим, что $G E\left(\bar{x}, \bar{g}^{t}\right)$ может иметь от двух до четырех концевых 
точек, и все эти точки лежат на абсолюте, хотя $G E\left(\bar{x}, \bar{g}^{t}\right)$ может иметь не более трех концевых точек в силу сверхтранзитивности потока $f^{t}$.

Шаг 7. Для любой точки $\bar{x} \in \bar{I}$ существуют открытый интервал $(\alpha(\bar{x}), \beta(\bar{x})) \subset \bar{C}$, содержащий точку $\bar{x}$, и окрестность $O^{1}(v, \bar{x}) \subset O_{1}^{1}(v) \subset$ $\chi^{1}(M)$ векторного поля $v$ такие, что концевъе точки $G E\left(\bar{x}^{\prime}, \bar{g}^{t}\right)$ лежат в $\delta$-окрестностях концевых точек $G E\left(\bar{x}, \bar{f}^{t}\right)$, где $\bar{x}^{\prime} \in(\alpha(\bar{x}), \beta(\bar{x}))$, а векторное nоле $w \in O^{1}(v, \bar{x})$ индуцирует поток $g^{t}$.

Согласно шагу 5 сушествуют поднятия $\bar{C}_{i}(\bar{x}), 1 \leqslant i \leqslant 3$, трансверсали $C$ такие, что концевые точки кривых $\bar{C}_{i}(\bar{x})$ ограничивают на абсолюте непересекающиеся дуги $\bar{d}_{i}(\bar{x}), 1 \leqslant i \leqslant 3$, каждая из которых содержит по одной концевой точке $G E\left(\bar{x}, \bar{f}^{t}\right)$, и каждая из которых имеет евклидову длину меньшую, чем $\delta$. Из теоремы о непрерывной зависимости компактных дуг траекторий от начальных условий $[13$, теорема 5, гл. 1$]$ и из теоремы об устойчивости сепаратрис гиперболических седел $[14$, теорема 6.2$]$ следует, что существуют открытый интервал $(\alpha(\bar{x}), \beta(\bar{x})) \ni \bar{x}$ и окрестность $O^{1}(v, \bar{x}) \subset O_{1}^{1}(v)$ векторного поля $v$ такие, что $G E\left(\bar{x}^{\prime}, \bar{g}^{t}\right)$ пересекает трансверсали $\bar{C}_{i}(\bar{x}), 1 \leqslant i \leqslant 3$, где поток $g^{t}$ индуцируется векторньм полем $w \in O^{1}(v, \bar{x})$. Но тогда концевые точки $G E\left(\bar{x}^{\prime}, \bar{g}^{t}\right)$ лежат в интервалах $\bar{d}_{i}(\bar{x})$, $1 \leqslant i \leqslant 3$. Отсюда следует требуемое утверждение.

Шаг 8. В силу компактности промељутка $\bar{I}$ существует конечное семейство интервалов $\left(\alpha\left(\bar{x}_{1}\right), \beta\left(\bar{x}_{1}\right)\right), \ldots,\left(\alpha\left(\bar{x}_{k}\right), \beta\left(\bar{x}_{k}\right)\right)$, покрывающих $\bar{I}$. Тогда окрестность

$$
O^{1}(v)=O^{1}\left(v, \bar{x}_{1}\right) \cap \cdots \cap O^{1}\left(v, \bar{x}_{k}\right)
$$

является искомой.

Согласно шагу 6 любое векторное поле $w \in O^{1}(v)$ индуцирует поток $g^{t} \in S_{g}^{t}$, имеющий глобальную секушую $C$. Отсюда следует, что поток $g^{t}$ имеет непустой геодезический каркас $G\left(g^{t}\right)$. Возьмем произвольную геодезическую $L^{\prime} \in G f\left(g^{t}\right)$ и докажем, что $L^{\prime} \in U$. Согласно определению геодезического каркаса любая накрывающая для $L^{\prime}$ геодезическая $\bar{L}^{\prime}$ является соответствующей геодезической для некоторой траектории или обобшенной траектории $\bar{l}^{\prime}$ ) накрываюшего потока $\bar{g}^{t}$, т.e. $\bar{L}^{\prime}=\bar{g}\left(\bar{l}^{\prime}\right)$.

Так как поток $g^{t}$ имеет глобальную секушую $C$, а отрезок $\bar{I}$ накрывает $C$, то, не уменьшая обшности, можно считать, что $\bar{l}^{\prime}$ ) пересекает $\bar{I}$ в некоторой точке, которую мы обозначим через $\bar{x}^{\prime}$. Тогда из шага 3 вытекает, что геодезическая $\bar{L}^{\prime}=\bar{g}\left(\bar{l}^{\prime}\right)$ пересекает геодезический сегмент $\bar{I}_{0}$.

Так как интервалы $\left(\alpha\left(\bar{x}_{1}\right), \beta\left(\bar{x}_{1}\right)\right), \ldots,\left(\alpha\left(\bar{x}_{k}\right), \beta\left(\bar{x}_{k}\right)\right)$ покрывают $\bar{I}$, то

$$
\bar{x}^{\prime} \in\left(\alpha\left(\bar{x}_{i}\right), \beta\left(\bar{x}_{i}\right)\right)
$$

для некоторого $1 \leqslant i \leqslant k$. В силу шага 7 концевые точки $G E\left(\bar{x}^{\prime}, \bar{g}^{t}\right)$ лежат в $\delta$-окрестностях концевых точек $G E\left(\bar{x}_{i}, \bar{f}^{t}\right)$. Поэтому существует траектория или обобщенная траектория $\bar{l}\left(\bar{x}_{i}\right)$ потока $\bar{f}^{t}$, проходящая через точку $\bar{x}_{i}$, такая, что концевые точки $\left.\bar{l}^{\prime}\right)$ и $\bar{l}\left(\bar{x}_{i}\right) \delta$-близки в евклидовой метрике на абсолюте. Отсюда вытекает, что геодезические $\bar{L}^{\prime}=\bar{g}\left(\bar{l}^{\prime}\right), \bar{g}\left(\bar{l}\left(\bar{x}_{i}\right)\right) \in \pi^{-1}\left(G\left(f^{t}\right)\right) \delta$-близки в евклидовом смысле. Из шага 4 тогда следует, что $\pi\left(\bar{g}\left(\bar{l}^{\prime}\right)\right) \in U$, т.е. $L^{\prime} \in U$. Это завершает доказательство теоремы 3.1. 
ДоКАЗАТЕЛЬСТво СЛЕДСТВИЯ 3.1. Согласно результатам работы [9] каждая геодезическая из $G\left(f^{t}\right)$ всюду плотна в $G\left(f^{t}\right)$. Поэтому каждая окрестность $U\left(L_{i}\right), i=1, \ldots, k$, является окрестностью геодезического каркаса $G\left(f^{t}\right)$. В силу теоремы 3.1 сушествует окрестность $O_{i}^{1}(v) \subset \chi^{1}(M)$ векторного поля $v$ такая, что любое векторное поле $w \in O_{i}^{1}(v)$ индуцирует поток $g^{t}$ с непустым геодезическим каркасом $G\left(g^{t}\right)$, который принадлежит $U\left(L_{i}\right), i=1, \ldots, k$. Искомая окрестность $O^{1}(v)$ есть $O^{1}(v)=O_{1}^{1}(v) \cap \cdots \cap O_{k}^{1}(v)$.

ДокаЗАТЕЛЬСТво ТЕОРемЫ 3.2. Известно, что векторные поля Морса-Смейла всюду плотны в пространстве $\chi^{1}(M)[14]$. Так как $f^{t} \in S_{g}^{t}$, то существует поток Морса-Смейла $g^{t}$, индуцируемьй векторньм полем $w \in O^{1}(v)$, такой, что точками покоя $g^{t}$ являются грубые седла. Согласно теореме 3.1 поле $w$ можно взять столь близким к $v$, что $G\left(g^{t}\right) \in U$. Так как в потоке Морса-Смейла нет нетривиально рекуррентных полутраекторий, то геодезический каркас $G\left(g^{t}\right)$ рациональный.

\section{§4. Два сценария разрушения рационального каркаса}

В основе доказательств теорем 4.1 и 4.2 лежит однопараметрическое семейство $C^{\infty}$-векторных полей в кольце $K_{1}$, лежащем на двумерной евклидовой плоскости и ограниченном двумя концентрическими окружностями $R_{1}, R_{3}$ с центром в начале координат. Будем считать, что в полярных координатах $(\rho, \varphi)$ окружность $R_{j}$, $j=1,3$, определяется уравнением $\rho=j$. Пусть $\vec{Q}_{0}$ - векторное поле, задающее динамическую систему $\dot{\varphi}=0, \dot{\rho}=1$. Начиная с $\vec{Q}_{0}$, можно построить на $K_{1}$ семейство $C^{\infty}$-векторных полей $\vec{Q}_{\mu}$, непрерьвно зависящих от параметра $\mu \in[0,1]$, со следующими свойствами:

- векторные поля $\vec{Q}_{\mu}, \mu \in[0,1]$, не имеют состояний равновесия в $K_{1}$ и совпадают с $\vec{Q}_{0}$ на окружностях $R_{1}, R_{3}$;

- траектории векторного поля $\vec{Q}_{\mu}, \mu \in[0,1)$, входят в кольцо $K_{1}$ под прямьгм углом через $R_{1}$ и выходят из $K_{1}$ под прямым углом через $R_{3}$;

- векторное поле $Q_{1}$ имеет один полуустойчивый цикл $E_{1}$, совпадающий как множество с окружностью $\rho=2$. Все остальные траектории при времени $t \rightarrow+\infty$ или $t \rightarrow-\infty$ навиваются на $E_{1}$.

Фактически семейство $\vec{Q}_{\mu}$ получается с помощью функционального поворота поля $\vec{Q}_{1}$, которое "уничтожает" полуустойчивый цикл $E_{1}$.

Обозначим через $-\vec{Q}_{\mu}$ векторное поле, противоположное полю $\vec{Q}_{\mu}$, и через $K_{2}-$ копию кольца $K_{1}$, на котором определено поле $-\vec{Q}_{\mu}$. Склеив кольца $K_{1}, K_{2}$ вдоль окружности $R_{3}$ с помошью тождественного преобразования, получим кольцо $K$, на котором векторные поля $\vec{Q}_{\mu},-\vec{Q}_{\mu}$ образуют $C^{\infty}$-векторное поле $\vec{F}_{\mu}$. Обе компоненты $C_{1}, C_{2}$ границы кольца $K$ гомеоморфны окружности $R_{1}$ и имеют естественную параметризацию, индуцируемую этой окружностью. Имея в виду эту параметризацию, сформулируем свойства семейства $\vec{F}_{\mu}$.

1) Траектории векторного поля $\vec{F}_{\mu}, \mu \in[0,1)$, индуцируют отображение последования Пуанкаре $C_{1} \rightarrow C_{2}$, которое не меняет параметра точек.

2) Векторное поле $\vec{F}_{1}$ имеет два полуустойчивых цикла, гомотопных кривым $C_{1}, C_{2}$ (см. рис. 2). 


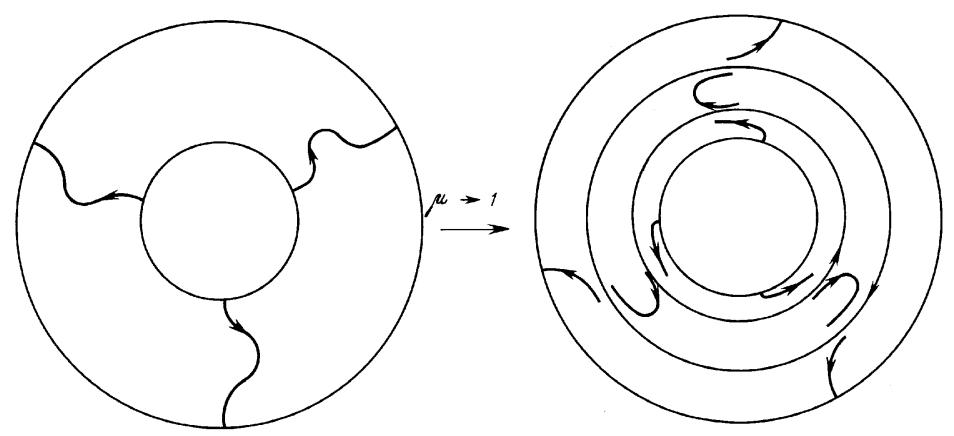

Рис. 2

Перейдем к доказательству теорем 4.1 и 4.2 .

ДоКАЗАТЕЛЬСТВо ТЕОРЕМЫ 4.1. Возьмем поток $f^{t}$ на поверхности $M$, который порождается $C^{\infty}$-векторным полем $\vec{V}$ и который является надстройкой над минимальньм перекладьванием открытых интервалов на окружности. В силу минимальности перекладьвания любая одномерная траектория потока $f^{t}$ всюду плотна на $M$. Так как $f^{t}$ является надстройкой, то $f^{t}$ имеет глобальную секущую $C$. Рассмотрим трубчатую окрестность $U$ секущей $C$, в которой поток $f^{t}$ топологически эквивалентен потоку поля $\vec{Q}_{0}$ на кольце $K_{1}$. Не уменшая общности, можно считать, что поле $\vec{V}$ ортогонально компонентам границы окрестности $U$. Заменим поле $\vec{V}$ на трубчатой окрестности $U$ векторным полем $\vec{F}_{\mu}, \mu \in[0,1]$. Полученное $C^{\infty}$-поле на $M$ обозначим через $\vec{V}_{\mu}$. Покажем, что семейство потоков $f_{\mu}^{t}$, индуцируемых векторными полями $\vec{V}_{\mu}, \mu \in[0,1]$, является искомым.

Действительно, так как любая одномерная траектория потока $f^{t}$ всюду плотна на $M$, то геодезический каркас $G\left(f^{t}\right)$ является неприводимым и иррациональньм. В силу условия 1$)$ на векторные поля $\vec{F}_{\mu}$, имеем, что геодезический каркас $G\left(f_{\mu}^{t}\right)=G\left(f^{t}\right)$ не зависит от параметра при $\mu \in[0,1)$. Согласно условию 2$)$ предельное множество потока $f_{1}^{t}$ состоит из двух полуустойчивых циклов $E_{1}, E_{2}$, гомотопных кривой $C$, и точек покоя. Поэтому геодезический каркас $G\left(f_{1}^{t}\right)$ потока $f_{1}^{t}$ рациональный и состоит из одной замкнутой геодезической $g$, гомотопной циклам $E_{1}, E_{2}$.

Так как полуустойчивые циклы $E_{1}, E_{2}$ гомотопны глобальной секущей $C$ потоков $f_{\mu}^{t}, \mu \in[0,1)$, то все геодезические каркаса $G\left(f_{\mu}^{t}\right)=G\left(f^{t}\right)$ трансверсально пересекают замкнутую геодезическую $g$. В силу замкнутости множества $G\left(f^{t}\right)$ угол пересечения геодезических из $G\left(f^{t}\right)$ с геодезической $g$ отделен от нуля положительной константой. Отсюда вытекает существование окрестности $U$ каркаса $G\left(f_{1}^{t}\right)=g$ такой, что $G\left(f_{\mu}^{t}\right) \notin U$ при $\mu \in[0,1)$.

ДОКАЗАТЕЛЬСТВО ТЕОРЕМЫ 4.2 полностью аналогично доказательству теоремы 4.1 , но в качестве исходного потока $f^{t}$ необходимо взять поток с непустым геодезическим каркасом, и который является надстройкой над перекладыванием открытых интервалов окружности. 


\section{§5. Бифуркации геодезических каркасов}

В этом параграфе мы докажем теоремы 5.1 и 5.2. В основе доказательства теоремы 5.1 лежит семейство $C^{\infty}$-векторных полей $\vec{R}_{\mu}, \mu \in[0,1]$, в кольце $K=$ $\{1 \leqslant \rho \leqslant 3,0 \leqslant \varphi \leqslant 2 \pi\}$, где $(\rho, \varphi)$ - полярные координаты на евклидовой плоскости. Используя семейство $C^{\infty}$-векторных полей $\vec{F}_{\mu}, \mu \in[0,1]$, построенное в $\S 4$, построим вначале семейство $\vec{R}_{\mu}, \mu \in\left[0, \frac{1}{2}\right]$, на кольце $K$ со следующими свойствами.

1) Векторные поля $\vec{R}_{\mu}, \mu \in\left[0, \frac{1}{2}\right]$, не имеют состояний равновесия в $K$ и перпендикулярны окружностям $R_{1}=\{\rho=1\}$ и $R_{3}=\{\rho=3\}$.

2) Траектории векторного поля $\vec{R}_{\mu}, \mu \in\left[0, \frac{1}{2}\right)$, входят в кольцо $K$ под прямьм углом через $R_{1}$ и выходят из $K$ под прямым углом через $R_{3}$. Более того, траектории поля $\vec{R}_{\mu}$ при $\mu \in\left[0, \frac{1}{2}\right)$ индуцируют отображение последования Пуанкаре $R_{1} \rightarrow R_{2}$, которое не меняет параметра $\varphi$.

3 ) Векторное поле $\vec{R}_{1 / 2}$ имеет два полуустойчивых цикла $E_{1}, E_{2}$, гомотопных $R_{1}, R_{2}$ (см. рис. 2).

Не уменьшая общности, можно считать, что $E_{1}, E_{2}$ являются двукратными предельными циклами (так как предельный полуустойчивый цикл $E_{1}$ поля $\vec{Q}_{1}$ можно взять двукратным, а семейство $\vec{F}_{\mu}$ построено на основании семейства векторных полей $\vec{Q}_{\mu}$, см. $\left.\S 4\right)$. Тогда один из этих циклов можно уничтожить с помощью функционального поворота векторного поля $\vec{R}_{1 / 2}$. Эту бифуркацию уничтожения одного из двукратных циклов обозначим через $\vec{R}_{\mu}, \mu \in\left[\frac{1}{2}, 1\right]$. Это завершает построение семейства векторных полей $\vec{R}_{\mu}, \mu \in[0,1]$, которое удовлетворяет еще одному условию (в добавок к выше перечисленным).

4) Векторное поле $\vec{R}_{1}$ имеет ровно один двукратный полуустойчивый цикл.

ДоКАЗАТЕЛЬСТво ТЕОРЕмЫ 5.1. Вначале мы построим специальное семейство потоков на двумерном торе $T^{2}$. Для замкнутой кривой $C$ на $T^{2}$ обозначим через $[C]$ длину геодезической, гомотопной $C$. Пусть $g_{0}^{t}-C^{\infty}$-поток на $T^{2}$ с единственным двукратньп полуустойчивьм циклом $l_{0}$, причем все остальные траектории потока $g_{0}^{t}$ являются двоякоасимптотическими к $l_{0}$ (отсюда, в частности, следует, что $l_{0}$ негомотопна нулю и $g_{0}^{t}$ не имеет точек покоя). В лемме 7 [12] доказано, что с помощью поворота векторного поля $\vec{V}_{0}$, который определяет поток $g_{0}^{t}$, можно получить последовательность потоков, у которых имеются пересекающие $l_{0}$ негомотопные нулю и негомотопные друг другу замкнутые траектории. Так как траектории этих полей трансверсальны траекториям потока $g_{0}^{t}$, то мы получаем, что $g_{0}^{t}$ имеет простую замкнутую трансверсаль $C_{0}$ такую, что $\left[C_{0}\right]>\left[l_{0}\right]$ (отметим, что в работе [12] более сильное утверждение сформулировано для аналитического потока $g_{0}^{t}$, однако необходимое нам следствие справедливо в классе гладкости $C^{\infty}$ ).

Сдеформировав, если это необходимо, поток $g_{0}^{t}$ в окрестности трансверсали $C_{0}$, можно получить трубчатую окрестность $U$ кривой $C_{0}$ такую, что траектории потока $g_{0}^{t}$ под прямым углом пересекают граничные компоненты окрестности $U$. Заменим в $U$ векторное поле $\vec{V}_{0}$ семейством векторных полей $\vec{R}_{\mu}, \mu \in[0,1]$. В результате мы получим семейство потоков $g_{\mu}^{t}$, задаваемых $C^{\infty}$-векторньпи полями $\vec{G}_{\mu}$, $\mu \in[0,1]$, такое, что 
- при каждом $\mu \in[0,1]$ предельное множество потока $g_{\mu}^{t}$ состоит либо из одного, либо из двух двусторонних предельных циклов;

- поток $g_{i}^{t}$ при $i=0$ и $i=1$ имеет единственный двукратный полуустойчивый цикл $l_{i}$, причем все остальные траектории потока $g_{i}^{t}$ являются двоякоасимптотическими к $l_{i}$;

- $\left[l_{0}\right]<\left[l_{1}\right]$.

Используя приведенную конструкцию, можно построить на торе семейство $C^{\infty}$-потоков $\hat{f}_{\mu}^{t}, \mu \in[0,1)$, непрерывно зависящих от параметра $\mu \in[0,1)$, такое, что при всех $\mu \in[0,1)$ поток $\hat{f}_{\mu}^{t}$ имеет один $E_{\mu}^{1}$ или два двусторонних полуустойчивых предельных цикла $E_{\mu}^{1}, E_{\mu}^{2}$, при этом длины геодезических, гомотопных $E_{\mu}^{1}, E_{\mu}^{2}$, равномерно стремятся к бесконечности при $\mu \rightarrow 1$. Так как в силу конструкции потоки $\hat{f}_{\mu}^{t}$ не имеют точек покоя, то можно считать, что сушествует такой открытый диск $D \subset T^{2}$, который непересекается с $\bigcup_{\mu}\left(E_{\mu}^{1} \cap E_{\mu}^{2}\right)$.

Возьмем точку $m_{0} \in D$ и ее окрестность $U\left(m_{0}\right) \subset D$. Для отрицательной полутраектории $l^{-}\left(m_{0}\right)$ точки $m_{0}$ произведем операцию Данжуа-Черри "раздувания" этой полутраектории потока $\hat{f}_{0}^{t}$ (наиболее общее описание этой операции с доказательством сушествования имеется в работе [3], см. также книгу [6]), т.е. в окрестности $U\left(m_{0}\right) \subset D$ появятся две точки покоя $m_{02}-$ устойчивый узел и $m_{01}-$ седло, связанные сепаратрисой $W_{2}^{u}\left(m_{01}\right)$ седла $m_{01}$. Полутраектория $l^{-}\left(m_{0}\right)$ преврашается в объединение сепаратрисы $W_{2}^{u}\left(m_{01}\right)$ и устойчивого многообразия $W^{s}\left(m_{02}\right)$ узла $m_{02}$. Отталкиваясь от $\hat{f}_{0}^{t}$, построим семейство, аналогичное $\hat{f}_{\mu}^{t}$ (которое мы также обозначим через $\left.\hat{f}_{\mu}^{t}\right)$. Поскольку диск $D$ не пересекается с $\bigcup_{\mu}\left(E_{\mu}^{1} \cap E_{\mu}^{2}\right)$, то полученное семейство потоков $\hat{f}_{\mu}^{t}$ по прежнему будет удовлетворять выше сформулированному свойству: при всех $\mu \in[0,1)$ поток $\hat{f}_{\mu}^{t}$ имеет один $E_{\mu}^{1}$ или два двусторонних полуустойчивых предельных цикла $E_{\mu}^{1}, E_{\mu}^{2}$, при этом $\left[E_{\mu}^{1}\right],\left[E_{\mu}^{2}\right] \rightarrow \infty$ при $\mu \rightarrow 1$.

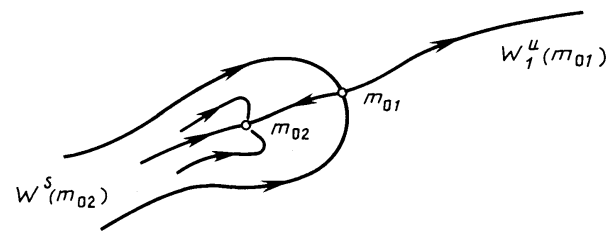

Рис. 3

Так как $m_{02}$ - узел, то у него сушествует окрестность $U\left(m_{02}\right) \subset W^{s}\left(m_{02}\right) \cup$ $W_{2}^{u}\left(m_{01}\right)$, диффеоморфная диску, такая, что граница $U\left(m_{02}\right)$ (диффеоморфная окружности) трансверсальна потокам $\hat{f}_{\mu}^{t}$. Удалим из тора окрестность $U\left(m_{02}\right)$ и склеим естественным образом два экземпляра $T^{2}-U\left(m_{02}\right)$, на одном из которых задано семейство векторных полей $\hat{V}_{\mu}$, индуцируюших потоки $\hat{f}_{\mu}^{t}$, а на другом - семейство векторных полей, противоположных полям $\hat{V}_{\mu}$. В результате мы получим замкнутую ориентированную поверхность $M_{2}$ рода 2 и семейство $C^{\infty}$-векторных полей на $M_{2}$, индуцируших семейство $C^{\infty}$-потоков $f_{\mu}^{t}$. 
В силу свойства $\left[E_{\mu}^{1}\right],\left[E_{\mu}^{2}\right] \rightarrow \infty(\mu \rightarrow 1)$ у потоков $\hat{f}_{\mu}^{t}$ построенное семейство удовлетворяет требованиям теоремы. Полностью аналогично строится требуемое семейство на замкнутой ориентированной поверхности любого рода.

ЗАмЕчАнИЕ. Если при доказательстве теоремы 5.1 каждую точку границы окрестности $U\left(m_{02}\right)$ сделать точкой покоя (т.е. "перешеек", соединяющий два тора, "перерезать" замкнутой кривой, заполненной точками покоя), то мы получим семейство потоков с рациональными геодезическими каркасами, состоящими только из замкнутых геодезических. Можно также потребовать, чтобы точки покоя потоков $f_{\mu}^{t}$ были гиперболическими седлами и узлами, расположенньми в фиксированных точках поверхности.

ДОКАЗАТЕЛЬСТВо ТЕОРЕМЫ 5.2. В силу условия теоремы при всех $\mu \in[0,1)$ поток $f_{\mu}^{t}$ имеет либо замкнутую негомотопную нулю траекторию $l_{\mu}$, либо замкнутый негомотопный нулю контур, состоящий из конечного числа точек покоя и сепаратрисных связей (который мы также обозначим через $l_{\mu}$ ). Более того, длины $\left[l_{\mu}\right]$ геодезических, гомотопных $l_{\mu}$, равномерно стремятся к бесконечности при $\mu \rightarrow 1$.

Предположим, что теорема неверна. Тогда существует точка $m_{0} \in M$, которая принадлежит топологическому пределу кривых $l_{\mu}$ и не является точкой покоя потока $f_{1}^{t}$. Так как $G\left(f_{1}^{t}\right)=\varnothing$, то положительная полутраектория $l^{+}\left(m_{0}\right)$ точки $m_{0}$ потока $f_{1}^{t}$ имеет предельное множество $\lim \left(l^{+}\left(m_{0}\right)\right)$ : либо гомотопную нулю замкнутую траекторию $l_{0}$, либо гомотопный нулю замкнутый контур, либо точку покоя $O$.

Отметим, что в силу теоремы о непрерывной зависимости компактных дуг траекторий от начальных условий и от возмушения потока (см. [15, теорема 8, гл. 2]), вся положительная полутраектория $l^{+}\left(m_{0}\right)$ принадлежит топологическому пределу кривых $l_{\mu}$.

Рассмотрим случай, когда $\lim \left(l^{+}\left(m_{0}\right)\right)=l_{0}$. Проведем через любую точку траектории $l_{0}$ отрезок без контакта $\Sigma$ и обозначим через $a, b \in \Sigma$ две последовательные (по времени) точки пересечения $l^{+}\left(m_{0}\right)$ с $\Sigma$. Тогда объединение отрезка $a b \subset \Sigma \mathrm{c}$ дугой траектории $l^{+}\left(m_{0}\right)$ между точками $a, b$ образует простую кривую $C$, которая ограничивает на поверхности односвязную область $D$. Не уменьшая общности, можно считать, что отрезок $\Sigma$ трансверсален потокам $f_{\mu}^{t}$ при всех $\mu \in[0,1]$. Так как точка $m_{0}$ принадлежит топологическому пределу кривых $l_{\mu}$, то кривые $l_{\mu}$ также пересекают отрезок без контакта $\Sigma$ в точках, близких к точкам $a, b$, и их дуги вместе с отрезком $\Sigma$ образуют замкнутые кривые, близкие к кривой $C$. Следовательно, кривые $l_{\mu}$ должны входить в некоторую односвязную область и не могут из нее выйти. Этого не может быть, поскольку кривые $l_{\mu}$ негомотопны нулю.

Полностью аналогично получаем противоречие в случае, когда $\lim \left(l^{+}\left(m_{0}\right)\right)$ есть гомотопный нулю замкнутый контур.

Рассмотрим случай, когда $\lim \left(l^{+}\left(m_{0}\right)\right)=O$ есть точка покоя потока $f_{1}^{t}$. Так как согласно предположению поток $f_{1}^{t}$ имеет конечное число точек покоя, то существует односвязная окрестность $U$ точки $O$, в которой нет других точек покоя, отличных от $O$. Не уменьшая общности, можно считать, что точка $m_{0}$ лежит вне окрестности $U$. Поскольку кривые $l_{\mu}$ негомотопны нулю, то они входят и выходят 
из окрестности $U$. Отсюда и из [16, теорема 60, гл. 8] вытекает, что положительная полутраектория $l^{+}\left(m_{0}\right)$ имеет продолжение по Бендиксону с той стороны, с которой кривые $l_{\mu}$ накапливаются к точке $m_{0}$. Другими словами, сушествует траектория $l^{1}$ потока $f_{1}^{t}$ такая, что ее $\alpha$-предельное множество совпадает с $O$, и $l^{1}$ принадлежит топологическому пределу кривых $l_{\mu}$. Снова из негомотопности нулю кривых $l_{\mu}$ следует, что можно считать, что траектория $l^{1}$ не стремится в положительном направлении к точке $O$. Повторяя предыдущие рассуждения, получим последовательность траекторий $l^{1}, \ldots, l^{k+1}=l^{1}$ таких, что $l^{i+1}$ является продолжением по Бендиксону траектории $l^{i}$ (с той стороны, с которой $l_{\mu}$ накапливаются к $\left.l^{i}\right), i=1, \ldots, k$. Тогда эта последовательность образует замкнутьй негомотопный нулю контур $K$, что противоречит условию $G\left(f_{1}^{t}\right)=\varnothing$, поскольку замкнутая геодезическая $g$, гомотопная контуру $K$, должна принадлежать геодезическому каркасу $G\left(f_{1}^{t}\right)$.

\section{Список литературы}

1. Anosov D. $V$. Flows on closed surfaces and behavior of trajectories lifted to the universal covering plane // J. Dyn. Control Syst. 1995. V. 1. № 1. P. 125-138.

2. Арансон C.X., Гринес В. З. О некоторых инвариантах динамических систем на двумерных многообразиях (необходимые и достаточные условия топологической эквивалентности транзитивных динамических систем) // Матем. сб. 1973. Т. 90. № 3. С. 372-402.

3. Gardiner $C$.J. The structure of flows exhibiting nontrivial recurrence on two-dimensional manifolds // J. Diff. Equat. 1985. V. 57. № 1. P. 138-158.

4. Аносов Д.В., Солодов В. В. Гиперболические множества. Аносов Д.В. и др. Динамические системы с гиперболическим поведением // Итоги науки и техн. Соврем. пробл. матем. Фундам. направления. Динамические системы - І. Т. 1. М.: ВИНИТИ, 1991. C. $12-99$.

5. Аносов Д. В. О поведении траекторий на плоскости Евклида или Лобачевского, накрывающих траектории потоков на замкнутых поверхностях, 1 // Изв. АН СССР. Сер. матем. 1987. Т. 51. №1. С. 16-43.

6. Aranson S., Belizky G., Zhuzhoma E. An Introduction to Qualitive Theory of Dynamical Systems on Surfaces. Amer. Math. Soc., Math. Monogr., 1996.

7. Арансон C.X., Гринес В.З. О представлении минимальных множеств потоков на двумерных многообразиях геодезическими линиями // Изв. АН СССР. Сер. матем. 1978. T. 42. № 1. C. 104-129.

8. Levitt G. Foliations and laminations on hyperbolic surfaces // Topology. 1983. V. 22. № 2 . P. 119-135.

9. Майер А. Г. О траекториях на ориентируемых поверхностях // Матем. сб. 1943. Т. 12. №1. C. 71-84.

10. Медведев В. С. О новом типе бифуркаций на многообразиях // Матем. сб. 1980. Т. 113. №3. C. 487-492.

11. Медведев В.С. О бифуркации "катастрофа голубого неба" на двумерных многообразиях // Матем. заметки. 1992. Т. 51. №1. С. 118-125.

12. Арансон $C . X$. Об отсутствии незамкнутых устойчивых по Пуассону полутраекторий и траекторий, двоякасимптотических к двойному предельному циклу, у динамических систем первой степени негрубости на ориентируемых двумерных многообразиях // Матем. сб. 1968. Т. 76. № 2. С. 214-230.

13. Баутин H. Н., Леонтович E. А. Методы и приемы качественного исследования динамических систем на плоскости. М.: Наука, 1990.

14. Palis J., de Melo J. W. Geometric Theory of Dynamical Systems. An Introduction. New York: Springer-Verlag, 1982. 
15. Андронов А.А., Леонтович Е.А., Гордон И.И., Майер А.Г. Теория бифуркаций динамических систем на плоскости. М.: Наука, 1967.

16. Андронов А.А., Леонтович Е.А., Гордон И. И., Майер А. Г. Качественная теория динамических систем второго порядка. М.: Наука, 1966.

Н. Новгород

Поступила в редакцию

27.10 .1996 